\title{
Uniportal video assisted thoracoscopy versus open surgery for pulmonary hydatid disease-a single center experience
}

\author{
Firas Abu Akar ${ }^{1}$, Diego Gonzalez-Rivas ${ }^{2,3}$, Bisanne Shaqqura ${ }^{1}$, Waseem Salman ${ }^{1}$, Mahmoud Ismail $^{4}$, \\ Tammah Safadi ${ }^{1}$, Rabee Adwan ${ }^{5}$, Haitham AL-Hassan ${ }^{6}$, Nisreen Rumman ${ }^{7}$, Nizar Hijjeh ${ }^{1}$ \\ ${ }^{1}$ Department of Cardiothoracic Surgery, Makassed Charitable Society Hospital, East Jerusalem, Palestine; ${ }^{2}$ Department of Thoracic Surgery, \\ Shanghai Pulmonary Hospital, Tongji University School of Medicine, Shanghai 200433, China; ${ }^{3}$ Department of Thoracic Surgery, Coruña \\ University Hospital, Coruña, Spain; ${ }^{4}$ Department of Thoracic Surgery, Klinikum Ernst von Bergmann Academic Hospital of the Charité - \\ Universitätsmedizin Humboldt University Berlin, Berlin, Germany; ${ }^{5}$ Infectious Diseases Unit, ${ }^{6}$ Division of Surgery, ${ }^{7}$ Department of Pediatrics, \\ Makassed Charitable Society Hospital, East Jerusalem, Palestine \\ Contributions: (I) Conception and design: F Abu Akar, B Shaqqura, N Rumman; (II) Administrative support: H Al-Hassan, N Hijjeh, T Safadi; \\ (III) Provision of study materials or patients: F Abu Akar, N Rumman, R Adwan; (IV) Collection and assembly of data: F Abu Akar, W Salman, B \\ Shaqqura; (V) Data analysis and interpretation: F Abu Akar; (VI) Manuscript writing: All authors; (VII) Final approval of manuscript: All authors. \\ Correspondence to: Firas Abu Akar, MD. Department of Cardiothoracic Surgery, Al-Makassed Charitable Society Hospital, East Jerusalem, Affiliated \\ to Al-Quds University School of Medicine, Jerusalem 97103, Palestine. Email: firasabuakar@gmail.com.
}

Background: Although rare in the Western world, the incidence of hydatid disease is still prevalent and strikingly endemic among the Palestinians. Until 2017, surgical treatment of lung pathologies was performed through the traditional incision (open thoracotomy). Uniportal video-assisted thoracoscopic surgery (VATS) approach has recently been applied in the cases of the pulmonary hydatid cysts with very satisfactory results.

Methods: Between January 2010 and January 2019, 39 patients with pulmonary HC disease have been surgically treated. The cases divided into two cohorts: operations performed by thoracotomy classified as group A, ( $n=16)$. Operations performed by uniportal VATS classified as group $B,(n=23)$. Prospectively collected data was analysed retrospectively, and the results compared between both groups.

Results: No significant statistical differences were noticed in terms of demographics and comorbidity. Laboratory tests were similar except haemoglobin level, which was higher in group A ( $\mathrm{P}=0.001)$. Despite that, blood transfusion was higher in group A $(\mathrm{P}=0.016)$. Moreover, operation time was longer in group A $(\mathrm{P}=0.000)$. Chest drainage remained longer in group A $(\mathrm{P}=0.077)$. The level of postoperative pain was significantly higher in group A certainly in POD $1(\mathrm{P}=0.000)$. Patients in group $\mathrm{B}$ discharged earlier from the hospital $(\mathrm{P}=0.011)$ and experienced lower complications $(\mathrm{P}=0.060)$. No significant difference in length of ICU stay. Neither recurrence nor 30-day mortality recorded in either group.

Conclusions: Uniportal VATS can be safely applied for pulmonary hydatidosis. It also seems to have a preference in several aspects compared to open Thoracotomy approach.

Keywords: Echinococcosis; uniportal video-assisted thoracoscopic surgery (uniportal VATS); pulmonary hydatid cyst; pulmonary parasitic infestation; intraoperative ultrasound

Submitted Jul 02, 2019. Accepted for publication Nov 19, 2019.

doi: $10.21037 /$ jtd.2019.12.73

View this article at: http://dx.doi.org/10.21037/jtd.2019.12.73

\section{Introduction}

Hydatid cyst disease is a zoonotic parasitic disease with high morbidity rate and socioeconomic impact in the endemic regions as in the Middle East, Eastern Europe and China (1). Its Annual costs estimated to be 3 billion US $\$$ for treatment and losses to the livestock industry as the WHO reported (2). The disease caused by metacestodes 
of Echinococcus granulosus. The parasite invades the human body and colonizes it after their eggs ingested through the digestive system (1). Four species of Echinococcus cause infection in humans. E. granulosus and E. multilocularis are the most common, while E. vogeli and E. oligarthrus, are less frequent (3). The parasite moves from the digestive system to the bloodstream, then settles in the body organs and forms vesicles. The liver is most commonly affected (75\%) followed by lungs (20\%), other organs are affected at varying rates (3). Single organ involvement occurs in $85 \%$ to $90 \%$ of cases, and single cyst observed in $70 \%$ of cases (4). Pulmonary hydatid cyst was first identified by Rudolf Virchow in 1855, since then, more Acknowledgements about the disease started to be well shaped (5). Records are the highest in South America, the Middle East, and Eastern Mediterranean, China, and the former Soviet Union. According to the WHO, in highly endemic regions, incidence rates in humans can exceed 50 per 100,000 person-year. The Mediterranean region where cystic echinococcus (CE) is endemical, Tunisia and Morocco reported the highest incidence of 12.5 and 5.1 surgical cases per 100,000, respectively (6). Individually, talking about Palestine, microbiologist series was published on 2017 reporting that $\mathrm{CE}$ found to be present in thirteen out of sixteen Palestinian districts. Al-Khalil (Hebron) and Bethlehem were the main foci of CE in Palestine with an incidence of 4.2 and 3.7 per 100,000 respectively. In some villages belonging to those provinces, the percentage was very high compared to the other governorates, as it reached, for example, 23.3 per 100,000 citizens in "AL-Shawawreh" area in Bethlehem (6). We also noted this relatively high incidence through our operative work. Surgical intervention is still considered to be the gold standard in the treatment of the pulmonary hydatid cysts once diagnosed. Parenchymal sparing surgery can be carried out in most cases without the need to resect a lung tissue, but this may be impossible in some cases, such as in consolidated cyst or in case of lobe destruction where lobectomy, segmentectomy or wedge resection should be considered (7-12). In our series, parenchymal-sparing operation was performed for the majority of the cases. Conservative medical treatment by albendazole used to be offered to patients with small cysts. However, complications as rupture, infection, and hemoptysis noted under conservative treatment, things that may be avoided with early surgery. In the last decade, there was a significant renaissance in thoracoscopic surgery. Several publications came up to describe conducting complex operations by uniportal VATS technique through the intercostal and subxiphoid approaches (13-17). Surgeons all over the world began to adapt and expertise these techniques for a wide variety of operations (18-24). After gaining experience in uniportal VATS by intensive training in Shanghai Pulmonary Hospital "Ultra-high volume VATS center" and mastering the technique in different types of pulmonary procedures (25), We started by adapting the method to endemic diseases in our region as pulmonary Hydatid cyst. We have found few publications in the literate describing the method of uniportal VATS as a surgical treatment of hydatid cyst (26). In this series, we are reporting 39 operated cases through the years [2010-2019] at Al-Makassed Hospital in East Jerusalem, comparing the cases that managed through the thoracotomy approach with the newly applied uniportal VATS.

\section{Methods}

We have retrospectively collected and reviewed data on surgical procedures for pulmonary HC conducted in our hospital between January 2010 and January 2019. In addition to clinical examination, chest X-rays and CT scans were the primary means for diagnosis. Serological test for echinococcus was unavailable in our clinic. However, we compared the demographic variables and the differences between the two groups in terms of perioperative related results. Statistical analysis was performed using the IBM SPSS [2011] statistics program for Windows (Version 23.0). Criterion for accepting a research hypothesis: Alpha $(\alpha)$ $=0.01$ (unilaterally). The criterion for negating preliminary differences between the groups (UVATS vs. thoracotomy): $\operatorname{Alpha}(\alpha)=0.05$ (bilaterally) For rational variables, means and standard deviations were calculated and baseline differences between the groups negated using a $t$-test. For nominal variables, absolute frequencies and percentages calculated, and baseline differences between the groups negated using Fisher's exact test. In order to test the differences between the groups (UVATS vs. thoracotomy) Mann and Whitney, $t$-tests and Fisher exact test conducted. All operations which performed before July 2017 were conducted by a single surgeon who adopts the open thoracotomy method while the rest were performed by another surgeon with experience in uniportal VATS surgeries. There were no criteria of exclusion in the UVATS group, including challenging cases (such as giant cysts, multiple or bilateral lesions, lobectomy or even pediatric cases). In our study, we describe the uniportal VATS technique in adults and pediatric patients and representing for the first time the ability to use the 
ultrasound for finding small and deep lesions.

\section{Surgical technique}

\section{Anesthesia considerations}

All the patients operated under general anesthesia. Double lumen tube intubation utilized for lung isolation in adult patients. However, in part of the pediatric patients (weighting $<30 \mathrm{~kg}$ ), single lumen tube was advanced into the main contralateral bronchus under the guidance of bronchoscope. Monitors attached for ECG, continuous invasive arterial blood pressure, end-tidal $\mathrm{CO}_{2}$, and $\left(\mathrm{SpO}_{2}\right)$. Intravenous (IV) access secured with large bore cannulas. IV hydrocortisone $100 \mathrm{mg}$ and IV chlorpheniramine $25 \mathrm{mg}$ was administrated before induction of anesthesia in anticipation of anaphylaxis. Adrenaline and Theophylline kept in standby for any emergencies. Lung isolation is crucial in this type of surgeries, as intraoperative lung manipulation could drain the contents of the cyst into the bronchial tree which can disseminate the disease, or causing anaphylaxis or acute airway obstruction. In addition to the importance of lungs isolation to conduct VATS operations, Lung isolation is crucial in this type of surgeries, as intraoperative lung manipulation could drain the contents of the cyst into the bronchial tree which can disseminate the disease, or causing anaphylaxis or acute airway obstruction. We have at no time utilized non-intubated VATS strategy in this type of surgeries as we belief that it may harm the safety of the patients. Three levels intercostal nerve block was performed at the end of the procedure under thoracoscopic vision utilizing a mixture of Lidocaine and Bupivacaine.

\section{Surgery}

UVATS

Lateral decubitus is the standard position. The elbow was fixed in 90 degrees (prayer's position) .A rolled sheet below the axilla is recommended to maximize intercostal space. We use a thoracoscope with $10 \mathrm{~mm}$ diameter $30^{\circ} \mathrm{HD}$ lens as a standard for adults while $5 \mathrm{~mm} 30^{\circ}$ used for pediatric patients. We start by careful exploring the pleural space for any ruptured cyst or free pleural membranes. In superficial cysts, the outermost layer of the cyst (the pericyst) is typically identified as a tens whitish layer with fine vascular nevi on its surface (Video 1). Identification of the small deep intra-parenchymal cyst is more challenging. Intraoperative ultrasound could be very helpful in this kind of cases when available (Video 2). After identifying the cyst, several pads soaked with hypertonic saline $(23.4 \%)$ are placed around the cyst to prevent contamination in case of rupture (Video 1). Using 21 gauge needle, the cyst wall is punctured and the fluid contents carefully aspirated until the cyst becomes collapsed (Video 1). Hypertonic saline "23.4\% concentration" injected through the same needle to refill the cyst. After waiting for 10-15 minutes, for the scolicidal effect of the saline, the fluid re-aspirated and the cyst opened using energy device. Cutting part of the wall edges improves the exposure and the closure of the cyst (Video 1). The cystic membrane is removed with precautions not to contact the patient's tissue (Figure 1). The cyst explored for additional membranes, then re-washed with hypertonic saline. Ventilation is done to check air-leaks through cyst-bronchial connections (This step is usually skipped in young pediatric patients who didn't underwent a double lumen intubation). The bronchial openings are closed one by one using 3-0 Vicryl sutures " $4-0$ in pediatrics" and capitonnage is done by plicating the cyst walls. In some cases, we inject BioGlue ${ }^{\circledR}$ (CryoLife Inc., Kennesaw, GA, USA) surgical adhesive inside the cyst to ensure hermetic closure. The pleural cavity is washed again with normal saline. The intercostal nerve block is done, and a chest tube inserted through the same incision before closing the wound.

\section{Open thoracotomy}

Positioning, draping and covering the patient is similar to the UVATS group, Posterolateral thoracotomy done. Rib spreader was inserted. Any adhesion between the cysts and the chest wall were lysed, pads soaked with hypertonic saline are applied before manipulating the cyst. Resection or capitonnage was performed by in the same manner as in the UVATS group. The wound closed in layers after placing 1-2 chest drains through additional incisions.

\section{Results}

\section{Baseline characteristics and comorbidities}

A total of 39 cases of pulmonary hydatid cysts retrospectively collected between January 2010 and January 2019. Overall, there were twenty-two male patients in both groups. The age of patients varied between 2 to 65 years (mean $=32.19$ and 31.39 years) in group A and group B respectively $(\mathrm{P}=0.894)$. Other demographic comparisons are shown in (Tables 1,2). The size of the cysts varies from one to eighteen $\mathrm{cm}$. The average diameter of the cysts in group A was $5.06 \mathrm{~cm}$ while the average diameter in group 

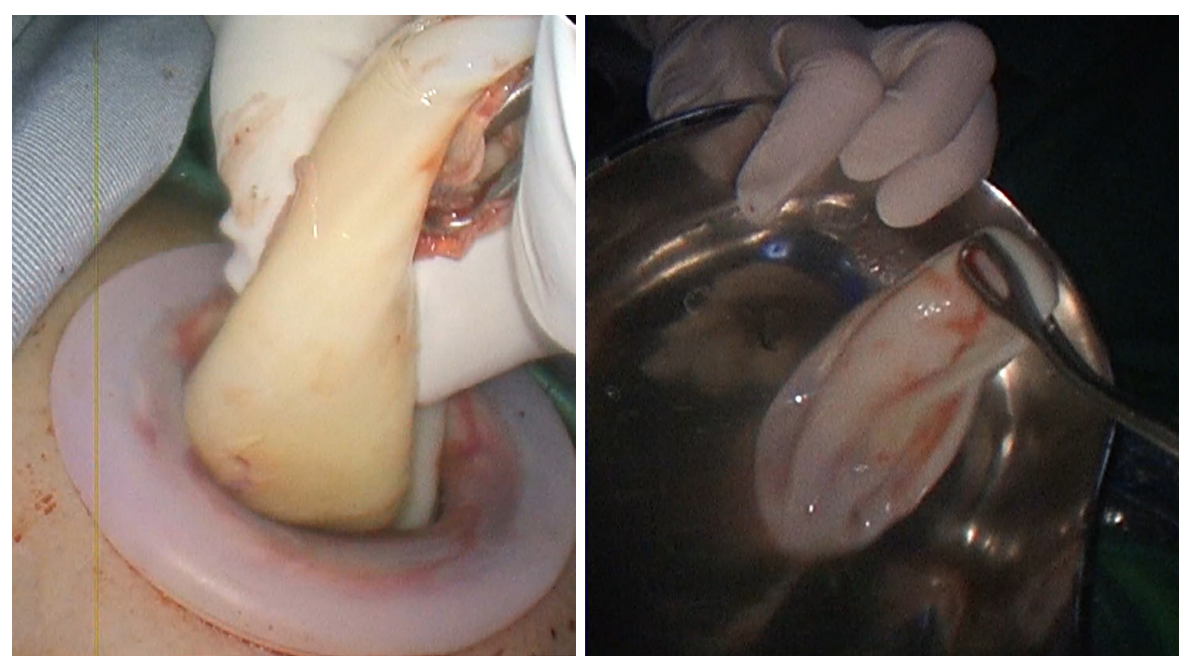

Figure 1 Germinative membrane after removal.

B was $5.78 \mathrm{~cm}$ with no significant statistical difference between the two groups. The number of cysts per patient ranged from one single cyst in most of the cases to up to five cysts (maximal number per one patient) in one patient who had bilateral disease. In this patient, a bilateral singlestaged UVATS surgery was carried out, and intra-operative ultrasound was utilized to find some deep and small lesions (Video 2). Figure 2 shows the geographical distribution of patients who came from different Palestinian cities. Seventy-one percent of the cases came from Bethlehem and Hebron cities, these findings consistent with the results of the study published by Al-Jawabreh and his colleagues (6). Preoperative rupture of the cyst was radiologically diagnosed in three patients (13\%) of the UVATS group "B" due to the "water lily sign" appearance in CT-scan (Figure 3). One of these patients arrived at the ER with unstable hemodynamic condition due to an anaphylactic reaction. He was admitted to the ICU and operated 48 hours later after stabilization of his condition. We could not obtain information on the number of ruptured cysts in the group "A" as that was not documented.

\section{Intra-operative and post-operative characteristics}

In total, Fifty-six pulmonary cysts operated in both groups. Right lower lobe was the most common location, followed by the left lower lobe. The middle lobe was the least prone to HC (Figure 4). In both groups, hepatic HC was present in seven patients (nine cysts in total), one other patient had a Pelvic HC and two other patients had a splenic
HC. One patient who had hepatic and pulmonary HC was treated by a single-staged combined laparoscopic and UVATS procedure, while the other cases were treated later by medications or surgery. Capitonnage was performed successfully in $63 \%$ of cases in group A vs. $87 \%$ in group B. In two cases of group B, lobectomy performed due to the solidification of the cyst. We want to point out that in these particular cases, the patient was under medical treatment with (Albendazole) for more than six months, which makes us wonder if there was a relationship between prolonged medical treatment and solidification of the cyst. In one case, a wedge resection was made for a small size cyst as we were not sure if it was a HC (confirmed later in the pathology report). The mean operative time was $167 \mathrm{~min}$ (range, 60-250 $\mathrm{min}$ ) in the open thoracotomy group "A" and $84 \mathrm{~min}$ (range, 25-210 min) in the UVATS group "B" $(\mathrm{P}=0.000)$. No conversions to multiportal VATS or thoracotomy recorded in group B. Intraoperative blood loss was significantly higher in group A $(\mathrm{P}=0.000)$. Although the level of blood hemoglobin was lower in group $\mathrm{B}$ when hospitalized $(\mathrm{P}=0.001)$, patients in this group required less packed $\mathrm{RBCs}$ and blood products transfusion because of the small proportion of blood loss during surgery compared to group A (Table 3). No difference in 30 days mortality rate for both groups $(0 \%)$. Overall complications rate was $44 \%$ in group A and $13 \%$ in group $\mathrm{B}(\mathrm{P}=0.060)$. Atelectasis and pneumonia were the most common complications in group A while surgical emphysema was the prominent complication in group B (Table 3). The amount of fluid discharged through the 
Table 1 Baseline characteristics and comorbidities

\begin{tabular}{|c|c|c|c|}
\hline Variables & Uniportal VATS (N=23) & Thoracotomy $(\mathrm{N}=16)$ & $P$ value \\
\hline Weight, mean (SD) & $73.70(19.29)$ & $65.93(15.67)$ & 0.212 \\
\hline Smoking, n (\%) & 9 [39] & $1[6]$ & 0.022 \\
\hline Cyst number in lungs, mean (SD) & $1.30(0.47)$ & $1.19(0.54)$ & 0.479 \\
\hline Operation type, n (\%)* & & & 0.043 \\
\hline Capitonnage & $20[87]$ & 10 [63] & \\
\hline Lobectomy & 2 [9] & $6[37]$ & \\
\hline Liver, n (\%) & $6[26]$ & 3 [19] & 0.446 \\
\hline HTN, n (\%) & $4[17]$ & $1[6]$ & 0.305 \\
\hline COPD, n (\%) & $0(0)$ & $0(0)$ & \\
\hline DVT, n (\%) & $0(0)$ & $0(0)$ & \\
\hline CAD, n (\%) & $0(0)$ & $0(0)$ & \\
\hline PVD, n (\%) & $0(0)$ & $0(0)$ & \\
\hline Surgical history, n (\%) & $5[22]$ & $7[44]$ & 0.133 \\
\hline
\end{tabular}

*, 1 from uniportal VATS had wedge resection operation. VATS, video-assisted thoracoscopic surgery; PFT, pulmonary function test; DM, diabetes mellitus; HTN, hypertension; COPD, chronic obstructive pulmonary disease; DVT, deep venous thrombosis; CAD, coronary artery disease; PVD, peripheral vascular disease.

Table 2 Admission clinical measures

\begin{tabular}{lccc}
\hline Variables & Uniportal VATS $(\mathrm{N}=23)$ & Thoracotomy $(\mathrm{N}=16)$ & $\mathrm{P}$ value \\
\hline Creatinine & $0.75(0.19)$ & $0.77(0.20)$ & 0.807 \\
WBC & $8,739[2,817]$ & $9,257[4,347]$ & 0.654 \\
WBC DOS & $12,713[2,940]$ & $13,001[4,954]$ & 0.827 \\
HGB & $9,630[3,452]$ & $14,102[3,866]$ & 0.001 \\
HGB dose & $13.05(2.28)$ & $12.68(2.11)$ & 0.607 \\
HGB POD 1 & $12.03(2.13)$ & $11.56(2.03)$ & 0.559 \\
Platelet & $11.70(1.94)$ & $11.50(1.62)$ & 0.736 \\
Platelet DOS & $277[79]$ & $273[97]$ & 0.888 \\
Platelet POD 1 & $227[81]$ & $277[105]$ & 0.108 \\
\hline
\end{tabular}

Continuous data are expressed as mean \pm SD. WBC, white blood cell; HGB, haemoglobin; POD, post operative day; VATS, video-assisted thoracoscopic surgery; DOS, day of surgery. 


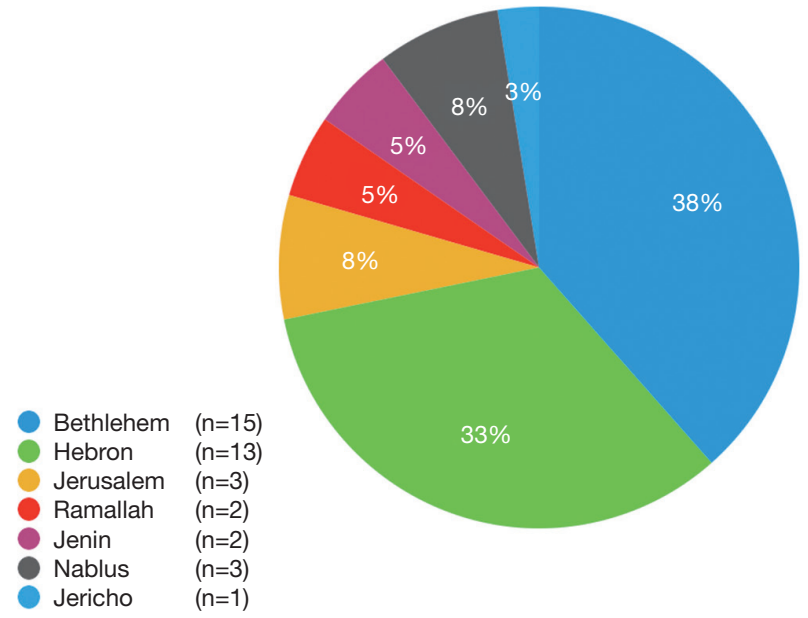

Figure 2 A diagram illustrates the geographic distribution of the patients.

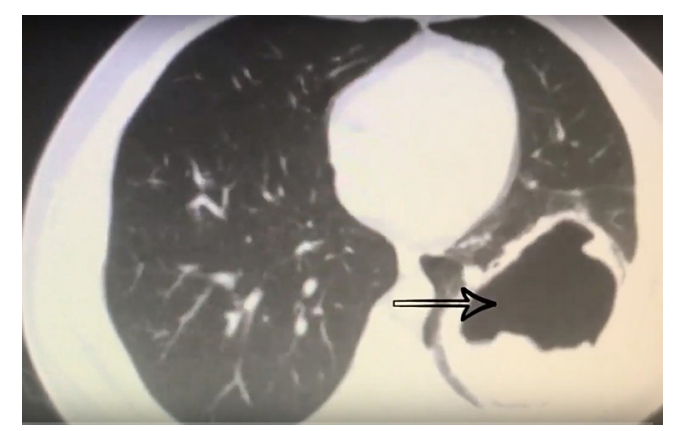

Figure 3 CT-scan showing ruptured hydatid cyst "Water-Lily sign".

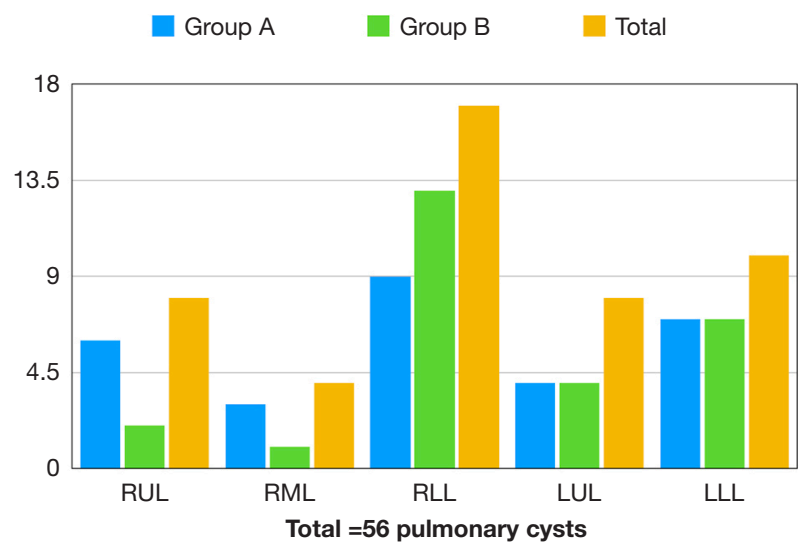

Figure 4 A diagram showing the distribution of the cysts among the pulmonary lobes and the rate of involvement of each lobe. RUL, right upper lobe; RML, right middle lobe; RLL, right lower lobe; LUL, left upper lobe; LLL, left lower lobe. chest drainage, and the amount of air leaks was significantly less among the patients in group $\mathrm{B}$, which resulted to faster removal of the chest drain $(\mathrm{P}=0.077)$ and earlier discharge from the hospital $(\mathrm{P}=0.011)$. We also analyzed the level of postoperative pain, which was examined according to the visual analog scale (VAS), this was assessed by the nurse three times per day. We compared the measurements taken in the morning $(6 \mathrm{am})$ in the first three days after the procedure, the results showed that pain was significantly lower in group B patients, especially in POD1 $(\mathrm{P}=0.000)$ (Figure 5). We should note that we did not compare the amount of pain medications given to the patients as the difference between them was great; hence we relied on the treatment for group A on strong narcotics as Morphine while in the group B, we were giving light painkillers as paracetamol and Dipyrone when needed.

\section{Discussion}

At a time when developed countries became almost free of the disease, Hydatid cyst disease is still considered a significant adult and pediatric health problem in the Palestinian territories due to its high incidence of up to $23 / 100,000$ in some areas (6). In addition to drug therapy, surgical intervention remains the most prominent and the gold standard in dealing with this disease. Despite the remarkable evolution of thoracoscopic surgery in the past two decades, the majority of surgeons are still adopting the traditional open thoracotomy approach for operating on pulmonary hydatid cysts. The reason for this may be the concern of not being able to control the contents of the cyst during the operation which could burst to the pleural cavity causing its contamination or anaphylactic response, so most surgeons resort to the way they consider "safer". However, some recent publications on thoracoscopic management of pulmonary hydatid cysts have been initiated in adults and pediatrics (27-29). As with other areas of thoracic surgery and as a natural progression of thoracoscopic techniques, these surgeries must have been performed by uniportal VATS (24,27-29). We believe that the presence of the high-definition thoracoscopic lens inside the thoracic cavity provides us very clear and accurate images in addition to focusing the image on the area of interest during the operation which may decrease the chance for the accidental rupture of the cyst. Additional advantage that the nurse and anaesthesiologist can follow the details of the surgery through the screen, which may 
Table 3 Outcome

\begin{tabular}{|c|c|c|c|}
\hline Variables & Uniportal VATS (N=23) & Thoracotomy $(\mathrm{N}=16)$ & $P$ value \\
\hline PRBCS units & $0.22(0.52)$ & $1.00(1.15)$ & $0.016^{A}$ \\
\hline FFP (cc) & 139 [273] & 263 [384] & $0.286^{A}$ \\
\hline Platelet units & $0.00(0.00)$ & $0.50(1.15)$ & $0.033^{A}$ \\
\hline Surgical blood loss & $131[76]$ & 401 [223] & 0.000 \\
\hline Surgery duration (min) & $84[47]$ & $167[54]$ & 0.000 \\
\hline Chest drain duration (days) & $1.65(0.83)$ & $6.94(11.14)$ & 0.077 \\
\hline Drainage amount day 1 (cc) & $90[70]$ & $261[124]$ & 0.00 \\
\hline Total drainage amount & 115 [108] & 523 [723] & 0.019 \\
\hline VAS day 1 & $3.61(1.62)$ & $6.13(1.45)$ & 0.000 \\
\hline VAS day 2 & $1.61(1.27)$ & $3.13(2.06)$ & 0.007 \\
\hline VAS day 3 & $1.33(1.15)$ & $2.69(2.02)$ & 0.048 \\
\hline ICU stay (days) & $2.78(4.44)$ & $2.75(1.94)$ & 0.978 \\
\hline Post-operative complications, $\mathrm{n}$ (\%) & $3[13]$ & $7[44]$ & 0.060 \\
\hline Air leak, n (\%) & $1[4]$ & $2[13]$ & 0.557 \\
\hline
\end{tabular}

Data are expressed as $\mathrm{n}$ (\%); continuous data are expressed as mean \pm SD. A, Mann and Whitney. PRBCS, Packed red blood cells; FFP, fresh frozen plasma; VAS, visual analog scale; ICU, intensive care unit.

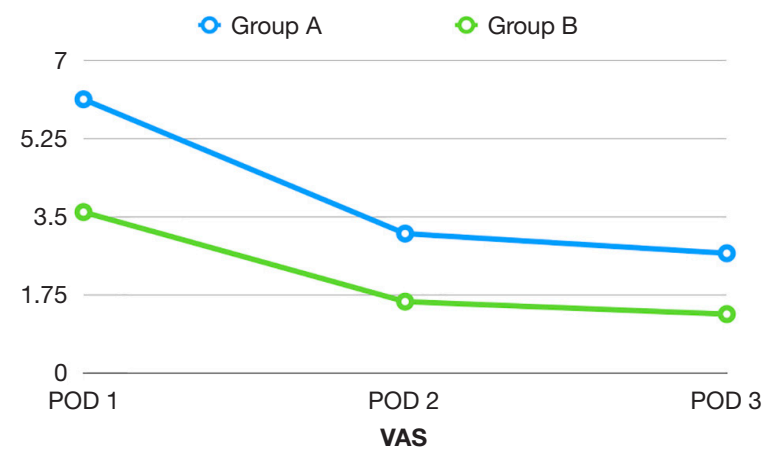

Figure 5 A diagram shows the difference in VAS scale between the two groups in the first three post-operative days. POD, post operative day; VAS, visual analog scale.

help them to take the appropriate action immediately in the event of a contingency order. Our cases were characterized by diversity, as the age for the patients varied "ranged between two years to sixty-seven years." The size of the cysts ranged from one $\mathrm{cm}$ to eighteen $\mathrm{cm}$. While most patients had a single cyst, some had multiple unilateral or bilateral cysts (up to five cysts). Due to the experience that we have gained in uniportal VATS technique (15-23), we have adopted this technique to conduct all the operations in the UVATS group without having any contraindication and without having to convert to open thoracotomy in any case. We had to perform a formal lobectomy in two cases due to the solid consistency of the lesion "chronic cyst," this may encourage earlier surgical intervention and prompts us not to postpone the procedure after diagnosis so that we may avoid lobectomy and preserve as much pulmonic tissue as possible. Undoubtedly, the limited amount of surgical trauma and pain provided by UVATS had stimulated us and given us the confidence to perform the single-stage bilateral operations when the cysts are present on both sides or combined thoracoscopic and laparoscopic procedures in cases of concomitant pulmonary and abdominal cysts. We always avoided doing this in open 
thoracotomy due to the anticipated pain, complications and exaggerated surgical trauma. Cysts which are distant from the pleural surface or tiny cysts may be a challenge for the surgeon to operate by UVATS due to the difficulty of localization by digital palpation. In such cases, we used the intraoperative ultrasound, this has dramatically helped us and replaced the issue of digital palpation, no matter how small the cyst was, no concern how far away from the surface. In conclusion, Uniportal VATS is a relatively new emerging technology that is continually evolving and gaining popularity among surgeons around the world. With the increased experience among the thoracoscopic surgeons, most complex and precise thoracic operations (including pulmonary $\mathrm{HC}$ ) are feasible to be performed safely. This study has been conducted in single-center, shows that the uniportal VATS technique, has several advantages over to the conventional open approach. We are aware that some of the limitations in this study are its retrospective nature, relatively small size of the cohort of patients in addition to the difference of surgeons in the two compared groups, which may cause some bias in the results. However, we hope that this study will be the basic for more substantial, prospective and multi-center studies in the future.

\section{Conclusions}

With the increased experience among the thoracoscopic surgeons, surgical treatment of pulmonary HC via Uniportal VATS technique became feasible to be performed safely. Intraoperative Ultrasound is Helpful for localizing small and deep lesions. Uniportal VATS technique, has several advantages over to the conventional open thoracotomy approach.

\section{Acknowledgments}

Funding: None.

\section{Footnote}

Conflicts of Interest: D Gonzalez-Rivas and M Ismail serve as the unpaid editorial board members of Fournal of Thoracic Disease from Dec 2019 to Nov 2021 and from Aug 2019 to Jul 2021 respectively. The other authors have no conflicts of interest to declare.

Ethical Statement: The authors are accountable for all aspects of the work in ensuring that questions related to the accuracy or integrity of any part of the work are appropriately investigated and resolved. The study was approved by the institutional review board at AL-Makassed Hospital.

Open Access Statement: This is an Open Access article distributed in accordance with the Creative Commons Attribution-NonCommercial-NoDerivs 4.0 International License (CC BY-NC-ND 4.0), which permits the noncommercial replication and distribution of the article with the strict proviso that no changes or edits are made and the original work is properly cited (including links to both the formal publication through the relevant DOI and the license). See: https://creativecommons.org/licenses/by-nc-nd/4.0/.

\section{References}

1. Bresson-Hadni SM, Mantion GA, Vuitton DA. Echinococcosis of the liver. In: Rodes JB, Blei AT, Reichen M, et al. editors. Textbook of hepatology. 3rd ed. Oxford: Blackwell Publishing, 2007:1047-57.

2. Budke CM, Deplazes P, Torgerson PR. Global socioeconomic impact of cystic echinococcosis. Emerg Infect Dis 2006;12:296-303.

3. McManus DP, Gray DJ, Zhang W, et al. Diagnosis, treatment, and management of echinococcosis BMJ 2012;344:e3866.

4. Agudelo Higuita NI, Brunetti E, McCloskey C. Cystic Echinococcosis. J Clin Microbiol 2016;54:518-23.

5. Tappe D, Frosch M. Rudolf Virchow and the recognition of alveolar echinococcosis, 1850s. Emerg Infect Dis 2007;13:732-5.

6. Al-Jawabreh A, Ereqat S, Dumaidi K, et al. The clinical burden of human cystic echinococcosis in Palestine, 20102015. PLoS Negl Trop Dis 2017;11:e005717.

7. Halezeroglu S, Celik M, Uysal A, et al. Giant hydatid cysts of the lung. J Thorac Cardiovasc Surg 1997;113:712-7.

8. Sarsam A. Surgery of pulmonary hydatid cysts. Review of 155 cases. J Thorac Cardiovasc Surg 1971;62:663-8

9. Doğan R, Yüksel M, Cetin G, et al. Surgical treatment of hydatid cysts of the lung: report on 1055 patients. Thorax 1989;44:192-9.

10. Xanthakis D, Efthimiadis M, Papadakis G, et al. Hydatid disease of the chest: report of 91 patients surgically treated. Thorax 1972;27:517-28.

11. Nahmias J, Goldsmith R, Soibelman M, et al. Three-to 7-year follow-up after albendazole treatment of 68 patients with cystic echinococcosis (hydatid disease). Ann Trop Med Parasitol 1994;88:295-304. 
12. Doğru D, Kiper N, Ozcelik U, et al. Medical treatment of pulmonary hydatid disease: for which child? Parasitol Int 2005;54:135-8.

13. Rocco G, Martucci N, La Manna C, et al. Ten-year experience on 644 patients undergoing single-port (uniportal) video-assisted thoracoscopic surgery. Ann Thorac Surg 2013;96:434-8.

14. Gonzalez D, Delgado M, Paradela M, et al. Uni-incisional video-assisted thoracoscopic left lower lobectomy in a patient with an incomplete fissure. Innovations (Phila) 2011;6:45-7.

15. Gonzalez-Rivas D, Paradela M, Fernandez R, et al. Uniportal video-assisted thoracoscopic lobectomy: two years of experience. Ann Thorac Surg 2013;95:426-32.

16. Hernandez-Arenas LA, Lin L, Yang Y, et al. Initial experience in uniportal subxiphoid video-assisted thoracoscopic surgery for major lung resections. Eur J Cardiothorac Surg 2016;50:1060-6.

17. Akar FA. Subxiphoid Uniportal Approach is it Just a Trend or the Future of VATS. MOJ Surg 2017;4:00076.

18. Gonzalez-Rivas D, Sihoe ADL. Important Technical Details During Uniportal Video-Assisted Thoracoscopic Major Resections. Thorac Surg Clin 2017;27:357-72.

19. Yang C, Abu Akar F, Chen J, et al. Right sleeve pneumonectomy via uniportal video-assisted thoracoscopic approach. J Thorac Dis 2018;10:E391-E396.

20. Abu Akar F, Yang C, Lin L, et al. Intra-pericardial double sleeve uniportal video-assisted thoracoscopic surgery left upper lobectomy. J Vis Surg 2017;3:51.

Cite this article as: Abu Akar F, Gonzalez-Rivas D, Shaqqura B, Salman W, Ismail M, Safadi T, Adwan R, AL-Hassan H, Rumman N, Hijjeh N. Uniportal video assisted thoracoscopy versus open surgery for pulmonary hydatid disease-a single center experience. J Thorac Dis 2020;12(3):794-802. doi: 10.21037/jtd.2019.12.73
21. Abu Akar F, Gonzalez-Rivas D, Fink D. Management of bleeding from an abnormally located S6 arterial branch in a common origin with S2 branch during live surgery. J Vis Surg 2016;2:87.

22. Abu Akar F, Gonzalez-Rivas D, Ismail M, Deeb M, et al. Uniportal video-assisted thoracic surgery: the Middle East experience. J Thorac Dis 2017;9:871-7.

23. Abu Akar FE, Zheng H, Ali G, et al. Bilateral uniportal video-assisted thoracoscopic lung resections. Interact Cardiovasc Thorac Surg 2017;25:1012-3.

24. Gonzalez-Rivas D, Lirio F, Sesma J, et al. Subxiphoid complex uniportal video-assisted major pulmonary resections. J Vis Surg 2017;3:93.

25. Abu Akar F, Gonzalez-Rivas D. Training in an ultra-highvolume center. Video-assist Thorac Surg 2018;3:17.

26. Ocakcioglu I, Sayir F. Uniportal Thoracoscopic Approach For Pulmonary Hydatid Cyst: Preliminary Results. Surg Laparosc Endosc Percutan Tech 2018;28:298-302.

27. Mallick MS, Al-Qahtani A, Al-Saadi MM, et al. Thoracoscopic treatment of pulmonary hydatid cyst in a child. J Pediatr Surg 2005;40:e35-7.

28. Parelkar SV, Gupta RK, Shah H, et al. Experience with video-assisted thoracoscopic removal of pulmonary hydatid cysts in children. J Pediatr Surg 2009;44:836-41.

29. Findikcioglu A, Karadayi S, Kilic D, et al. Video-assisted thoracoscopic surgery to treat hydatid disease of the thorax in adults: is it feasible? J Laparoendosc Adv Surg Tech A 2012;22:882-5. 